\title{
EASY-SRA: Um sistema de resposta à audiência para avaliação contínua
}

\author{
Álvaro Francisco de Castro Medeiros \\ Universidade Federal da Paraíba - PPGI - alvaro@di.ufpb.br \\ Thiago Henrique Jacob Oliveira Sousa \\ Universidade Federal da Paraíba - PPGI - thiago.henrique.jacob@gmail.com \\ Ed Porto Bezerra \\ Universidade Federal da Paraíba - PPGI - edporto@di.ufpb.br \\ Jarbele Cássia da Silva \\ Universidade Federal da Paraíba - PPGI - jarbele.cassia@dce.ufpb.br
}

\begin{abstract}
Resumo - Sistemas de Resposta à Audiência (SRA) tem sido utilizados como uma alternativa para obter o contínuo feedback dos alunos em sala de aula, bem como auxiliar o modelo de ensino/aprendizagem no ambiente eletrônico e online. Neste artigo é apresentada uma análise de diferentes SRA com ênfase em suas principais funcionalidades. Seu propósito consiste em, a partir das funcionalidades registradas, propor um novo sistema SRA, o EASY-SRA, cujo diferencial está na otimização para dispositivos móveis, disponibilidade em diferentes idiomas, diferentes possibilidades de votação, integração com redes sociais, entre outras características elencadas neste estudo.
\end{abstract}

Palavras Chaves - sistemas de resposta à audiência (SRA), modelagem conceitual, EASY-SRA.

\section{EASY-SRA: An audience response system for the continuous assessment}

\begin{abstract}
Audience Response Systems (ARS) has been used as an alternative to get continuous feedback from students in the classroom, as well as assisting the model of teaching / learning in the electronic environment and online. This article presents an analysis of different ARS with emphasis on its main features. Its purpose is to, from the registered features, proposing a new ARS system, the EASY-SRA, whose differential is the optimization for mobile devices, available in different languages, different voting possibilities, integration with social networks, among other characteristics listed in this study.
\end{abstract}

Keywords - audience response systems (ARS), conceptual modeling, EASY-SRA.

\section{Introdução}

O uso de subsídios computacionais têm se tornado uma alternativa cada vez mais constante no processo de ensino e aprendizagem em diversas áreas do conhecimento. Tanto em escolas como em universidades, estas práticas têm emergido e sido utilizadas em auxílio aos exercícios da aprendizagem.

O uso de tecnologias na educação contemporânea esta em evidência. Dentre estas tecnologias estão softwares educativos, equipamentos eletrônicos e/ou computadores. 
Também com a acessibilidade a web e aos dispositivos móveis, os alunos se sentem motivados a aprenderem os conteúdos acadêmicos de maneira mais fácil, rápida e divertida.

O aprendizado eletrônico caracteriza-se pela execução de atividades não presenciais com auxílio de meios eletrônicos. O grande problema destes ambientes é manter a motivação e o monitoramento do corpo discente. É neste contexto que surgem os Sistemas de Resposta à Audiência (SRA, que é uma abreviação portuguesa para o termo em Inglês de Audience Response Systems). Um SRA é utilizado como uma alternativa para obter o contínuo feedback dos alunos, inteirando-os ainda mais nas práticas desenvolvidas na sala de aula e avaliando os resultados gerados a partir da aprendizagem obtida (Gauci, et. al. 2009) (Beatty, et. al. 2006) (Mantoro, et. al. 2010).

A proposta deste estudo é discutir e avaliar o uso da abordagem dos SRA objetivando o desenvolvimento de um novo sistema SRA, chamado EASY-SRA.

Este artigo apresenta a seguinte estrutura: a Seção 2 discute os Sistemas SRA; a Seção 3 aborda a modelagem conceitual do sistema EASY-SRA, enfatizando todo o trabalho realizado; a Seção 4 trata das considerações finais sobre o estudo e de suas perspectivas futuras.

\section{Sistemas de Resposta à Audiência}

SRA são tanto hardwares quanto softwares que permitem estudantes de cursos presenciais ou à distância responderem questões utilizando um aparelho de controle remoto. Estes permitem que alunos respondam a questões mostradas em uma tela clicando em seus dispositivos remotos (Kay e Lesage, 2009). Após os alunos responderem uma votação, os resultados são apresentados para a classe em um formato visual, usualmente um histograma. A Figura 1 demonstra um resultado de uma votação do SRA Survnvote (Mantoro, et. al. 2010), onde a opção B foi a mais votada.

As respostas são coletadas através de dispositivos chamados clicadores os quais são pequenos transmissores do tamanho de um controle remoto de televisão onde estudantes respondem questões apertando botões (Caldwell, 2007). A Figura 2 mostra um exemplo de clicador.

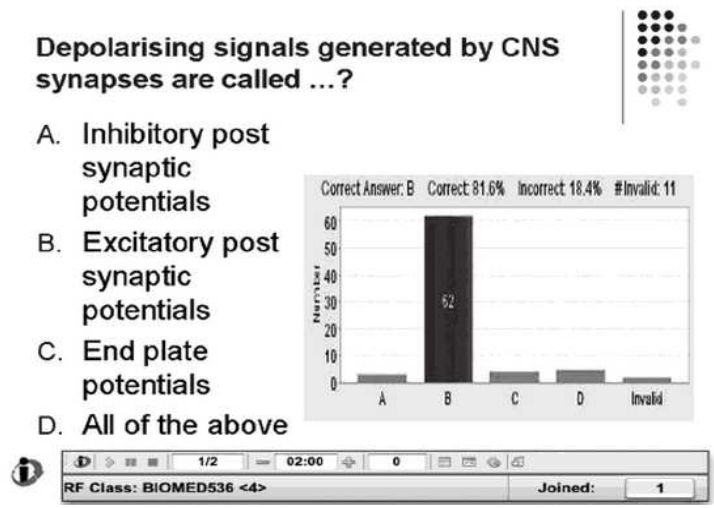

Figura 1 - Exemplo de resultado de votação do SRA Survnvote

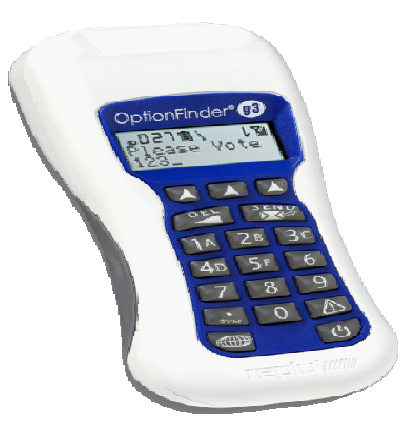

Figura 2 - Exemplo de clicador

SRA permitem que estudantes respondam a questões anonimamente ou que professores rastreiem através do aparelho de controle remoto para saber de qual 
estudante veio às respostas. SRA dão feedback contínuo para os estudantes e para o professor sobre o desempenho da classe.

Vários estudos têm demonstrado os benefícios da utilização de SRA para obter maior engajamento dos alunos e melhores resultados de aprendizagem (Gauci, et. al. 2009) (Beatty, et. al. 2006) (Mantoro, et. al. 2010) (Kay e Lesage, 2009) (Caldwell, 2007).

\section{EASY-SRA}

Realizou-se como primeiro passo do processo de elaboração do EASY-SRA uma análise de outros SRA para identificar suas funcionalidades para que o EASY-SRA incorpore as principais. Além destas, funcionalidades adicionais foram identificadas. A metodologia de análise e a modelagem conceitual do EASY-SRA são apresentados nesta seção.

\subsection{Metodologia de análise}

Definiu-se a metodologia utilizada para a análise dos SRA numa sequência de três passos. No primeiro passo, realizaram-se buscas em artigos de bases de dados online de periódicos para catalogar SRA. No segundo passo, as características gerais de cada um destes foram registradas. No terceiro e último passo, manipulou-se cada um dos sistemas para conhecer suas funcionalidades e assim criar uma lista preliminar de requisitos.

Utilizando-se desta metodologia pode-se levantar e registrar uma grande gama de requisitos preliminares e outros requisitos não atendidos por estes sistemas que seriam passíveis de serem adicionados ao sistema EASY-SRA.

A Tabela 1 apresenta o resultado das atividades realizadas no primeiro e segundo passo, onde as funcionalidades das ferramentas SRA foram consideradas.

Tabela 1 - Análise de sitemas SRA

\begin{tabular}{|c|c|c|c|c|c|c|c|}
\hline Característica & 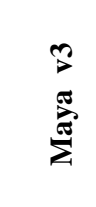 & 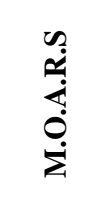 & 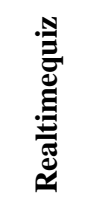 & 总 & 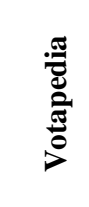 & 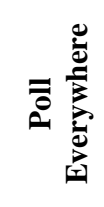 & 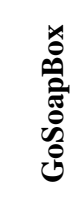 \\
\hline \multicolumn{8}{|l|}{ Open-source } \\
\hline \multicolumn{8}{|l|}{ Gratuito } \\
\hline Linguagem & PHP & PHP & PHP & PHP & PHP & & \\
\hline \multicolumn{8}{|l|}{$\begin{array}{l}\text { Integração ao } \\
\text { MOODLE }\end{array}$} \\
\hline Formas de responder & $\begin{array}{c}\text { PC } \\
\text { Smart } \\
\text { phone } \\
\text { SMS }\end{array}$ & $\begin{array}{l}\text { PC } \\
\text { Smart } \\
\text { phone }\end{array}$ & $\mathrm{PC}$ & $\begin{array}{c}\text { PC } \\
\text { Smart } \\
\text { phone } \\
\text { SMS }\end{array}$ & $\begin{array}{l}\text { PC } \\
\text { Smart } \\
\text { phone }\end{array}$ & $\begin{array}{l}\text { PC } \\
\text { Smart } \\
\text { phone } \\
\text { SMS }\end{array}$ & $\begin{array}{c}\text { PC } \\
\text { Smart } \\
\text { phone } \\
\text { SMS }\end{array}$ \\
\hline $\begin{array}{l}\text { Suporte a outros } \\
\text { idiomas }\end{array}$ & & & $\begin{array}{l}\text { Inglês } \\
\text { Alemão }\end{array}$ & Inglês & & & \\
\hline \multicolumn{8}{|l|}{ Documentação } \\
\hline Otimização de interface & $\begin{array}{l}\text { iPhone } \\
\text { iPad }\end{array}$ & & & & & & \\
\hline
\end{tabular}


As características open-source e gratuidade não estão presentes no Poll Everywhere e GoSoapBox. Também não pode ser identificada a linguagem de programação utilizada nestes, por não permitirem acesso ao código-fonte. Nenhum dos SRA apresentou integração a rede social Facebook. A Tabela 2 apresenta o resultado das atividades realizadas no terceiro passo.

Tabela 2 - Requisitos Preliminares

\begin{tabular}{|c|c|}
\hline Requisito preliminar & Descrição \\
\hline Requisto Preliminar 01 & $\begin{array}{l}\text { visualização da lista de disciplinas cadastradas por ele } \\
\text { anteriormente no início da utilização do sistema. }\end{array}$ \\
\hline Requisito Preliminar 02 & cadastro de uma nova disciplina. \\
\hline Requisito Preliminar 03 & $\begin{array}{l}\text { edição de dados de uma disciplina previamente cadastrada } \\
\text { por este. }\end{array}$ \\
\hline Requisito Preliminar 04 & exclusão de uma disciplina previamente cadastrada por este. \\
\hline Requisito Preliminar 05 & $\begin{array}{l}\text { visualização da lista de alunos vinculados a uma disciplina } \\
\text { previamente cadastrada por este. }\end{array}$ \\
\hline Requisito Preliminar 06 & $\begin{array}{l}\text { adição e remoção de outros usuários com perfis de alunos } \\
\text { de uma disciplina criada previamente por este. }\end{array}$ \\
\hline Requisito Preliminar 07 & $\begin{array}{l}\text { visualização da lista das votações criadas por ele para uma } \\
\text { determinada disciplina. }\end{array}$ \\
\hline Requisito Preliminar 08 & cadastro de novas votações em uma turma. \\
\hline Requisito Preliminar 09 & edição de uma votação selecionada. \\
\hline Requisito Preliminar 10 & $\begin{array}{l}\text { exclusão de uma votação selecionada. O sistema solicitará a } \\
\text { confirmação do usuário antes de realizar a exclusão. }\end{array}$ \\
\hline Requisito Preliminar 11 & iniciação de uma votação selecionada em uma disciplina. \\
\hline Requisito Preliminar 12 & $\begin{array}{l}\text { mudança de votação ativa sem necessidade de retornar a } \\
\text { listagem de votaçôes. }\end{array}$ \\
\hline Requisito Preliminar 13 & $\begin{array}{l}\text { O sistema deve identificar de qual ente da audiência cada } \\
\text { resposta esta sendo submetida e de que tipo de dispositivo } \\
\text { este a enviou (computador ou smartphone). }\end{array}$ \\
\hline Requisito Preliminar 14 & $\begin{array}{l}\text { realização de download da votação em formato de slide } \\
\text { compatível com os principais softwares de apresentação do } \\
\text { mercado. }\end{array}$ \\
\hline Requisito Preliminar 15 & envio da votação por ativa por email. \\
\hline
\end{tabular}

Como resultado destas análises, identificaram-se as seguintes características para o sistema EASY-SRA:

- Possuir um website funcional.

- Ser uma aplicação web e possuir uma versão em forma de plugin para o Moodle.

- Ser open-source.

- Ser gratuito.

- Ser construído com a utilização da linguagem de programação PHP e do paradigma de programação orientada a objetos.

- Ser integrável ao Facebook.

- Permitir que as votações sejam respondidas através do computador, smartphone ou SMS.

- Possuir suporte aos idiomas português, inglês e espanhol. 
- Possuir uma documentação escrita nos idiomas português, inglês e espanhol.

- Possuir otimizações de interface para todos os dispositivos móveis.

\subsection{Modelagem Conceitual}

Classificaram-se os requisitos preliminares em duas categorias: requisitos funcionais (Tabela 3) e requisitos não funcionais (Tabela 4) (Sommervile, 2007).

Tabela 3 - Exemplo de Requisito Funcional do EASY-SRA

\begin{tabular}{|c|c|}
\hline \multicolumn{2}{|c|}{ RF-001 Visualizar lista de disciplinas } \\
\hline Atributo & Descrição do atributo \\
\hline Nome: & Visualizar lista de disciplinas \\
\hline Descrição: & $\begin{array}{l}\text { O sistema deve permitir que um usuário } \\
\text { visualize a lista de disciplinas cadastradas } \\
\text { por ele anteriormente no início da utilização } \\
\text { do sistema. Os dados que deverão ser } \\
\text { demonstrados são o ano/semestre da } \\
\text { disciplina, nome, descrição, data de início e } \\
\text { data de fim e imagem em miniatura } \\
\text { vinculada a disciplina. }\end{array}$ \\
\hline Requisitos Funcionais Associados: & Nenhum requisito funcional associado. \\
\hline Requisitos Não Funcionais Associados: & $\begin{array}{l}\text { RNF-003 - Controle de acesso } \\
\text { Pré-condição: O requisito só pode ser } \\
\text { acessado por um usuário previamente } \\
\text { autorizado com o perfil de professor. }\end{array}$ \\
\hline
\end{tabular}

Tabela 4 - Exemplo de Requisito Não-Funcional do EASY-SRA

\begin{tabular}{|l|l|}
\hline \multicolumn{2}{|c|}{ RNF-001 - Interoperabilidade com o Moodle } \\
\hline Atributo & Descrição do atributo \\
\hline Nome: & Interoperabilidade com o Moodle \\
\hline Descrição: & $\begin{array}{l}\text { O sistema deve ser capaz de se integrar ao } \\
\text { sistema gerenciador de aprendizagem } \\
\text { Moodle como uma ferramenta externa ou um } \\
\text { plugin acoplável }\end{array}$ \\
\hline Categoria: & Funcionais \\
\hline $\begin{array}{l}\text { Requisitos } \\
\text { Associados: }\end{array}$ & $\begin{array}{l}\text { RNF-003 - Controle de acesso } \\
\text { Pré-condição: O requisito só pode ser } \\
\text { acessado por um usuário previamente } \\
\text { autorizado com o perfil de professor. }\end{array}$ \\
\hline
\end{tabular}

Os casos de uso permitem que se tenha uma visão do sistema do ponto de vista do usuário, facilitando assim a criação do sistema. Como primeira atividade realizada para a elaboração destes, definiram-se os seguintes atores que interagiriam com o sistema:

- Professor: Gerenciará as votações que serão utilizadas em sala de aula.

- Professor Administrador: realizará todas as ações que um professor realiza e ainda poderá gerenciar os usuários cadastrados. 


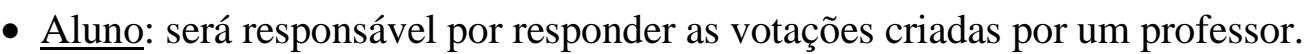

Após a definição dos Atores, partiu-se para a elaboração dos diagramas de casos de uso.

O primeiro diagrama de casos de uso elaborado (Figura 3) foi o para todos os Atores, o qual representa as ações que serão realizadas por todos os Atores que interagem com o sistema EASY-SRA. As ações que poderão ser realizadas por todos os Atores são aquelas mais genéricas e independentes de perfil, tais como realizar login, redefinir a própria senha, cadastrar um novo usuário e editar os dados do usuário.

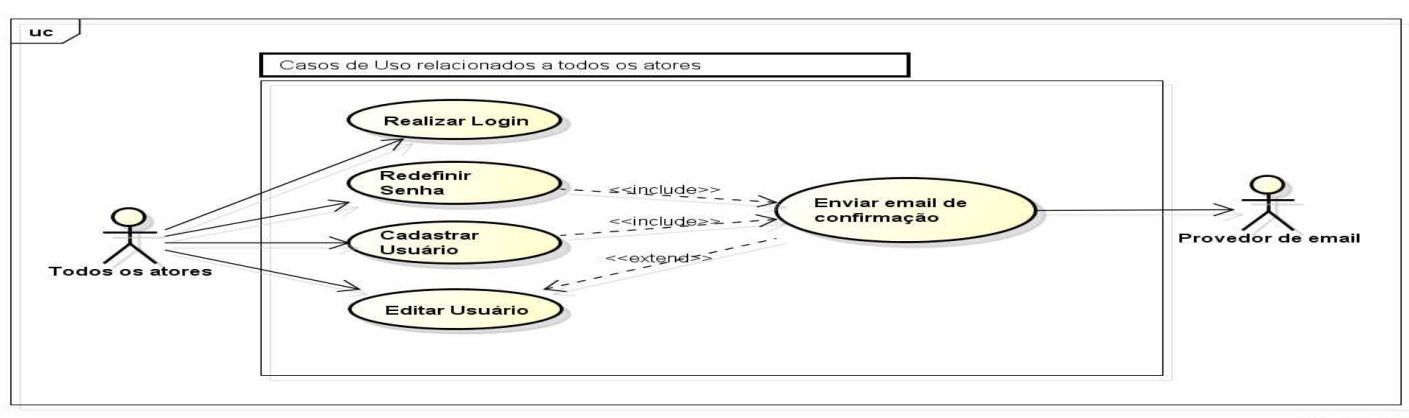

Figura 3 - Casos de uso para o todos os Atores

O segundo diagrama de casos de uso elaborado (Figura 4) foi para o Ator Professor. A maior parte das ações que podem ser realizadas no sistema EASY-SRA são feitas pelo ator Professor. Este pode gerenciar disciplinas(visualizar, cadastrar, editar e excluir), gerenciar alunos vinculados a uma disciplina (visualizar, adicionar e excluir), gerenciar votações vinculadas a uma disciplina (visualizar, cadastrar, editar e excluir) e outras ações relacionas a votação.

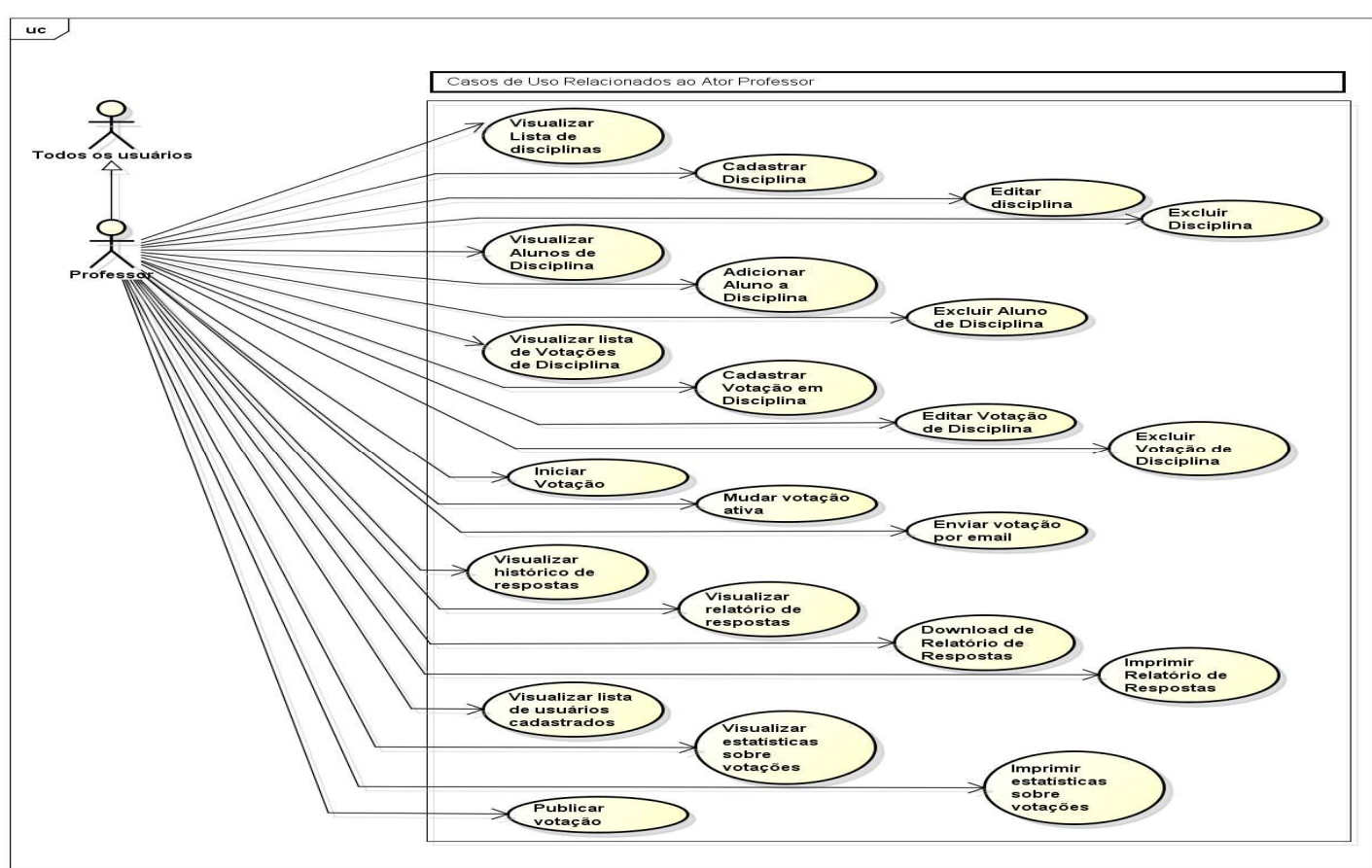

Figura 4 - Casos de uso para o Ator professor 
O terceiro diagrama de casos de uso elaborado (Figura 5) foi para o Ator Professor Administrador. Este pode realizar todas as ações do ator Professor e ainda algumas ações administrativas como excluir usuários, visualizar solicitações de cadastro de novos usuários e autorizar solicitações de cadastro de novos usuários.

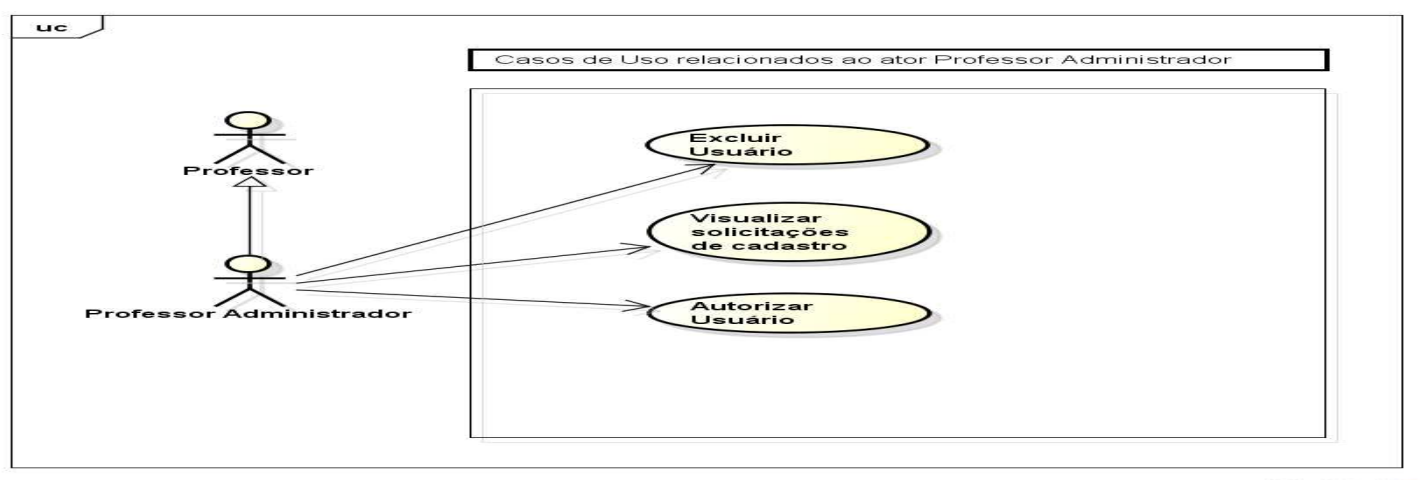

Figura 5 - Casos de uso para o Ator Professor Administrador

O quarto, e último, diagrama de casos de uso elaborado (Figura 6) foi para o Ator Aluno. Este pode visualizar todas a votações iniciadas e vinculadas a disciplina que este participa e responder a votações.

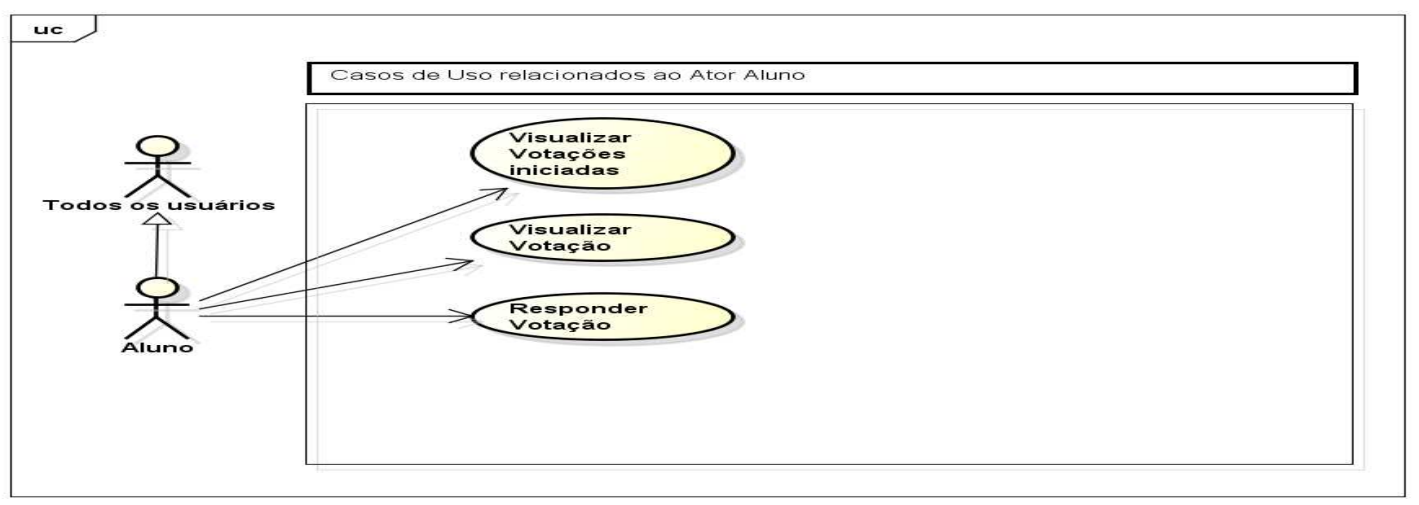

Figura 6 - Casos de uso para o ator Aluno

Foram identificadas, para elaboração do modelo relacional da base de dados, as tabelas users, disciplines, registrationrequests, profiles, sessions, emails, images, polls, votes e answers.

A tabela users conterá dados dos usuários registrados no sistema. A tabela disciplines armazernará dados das disciplinas registradas por usuários. A tabela registrationrequests possuirá dados de solicitações de cadastro feitas ao sistema. A tabela profiles armazernará dados relativos aos três perfis de usuário que podem acessar sistema (professor, professor administrador, aluno). A tabela sessions conterá das sessões de usuário. A tabela emails possuirá dados dos emails enviados por usuário ao sistema. A tabela images guardará dados das imagens registradas para votações ou disciplinas. A tabela polls armazernará dados das votações registradas para cada disciplina. A tabela votes guardará dados dos votos submetidos para cada votação e a tabela answers armazernará dados das respostas vinculadas a cada voto submetido a uma votação. A Figura 7 mostra o modelo relacional da base de dados dos EASY-SRA. 


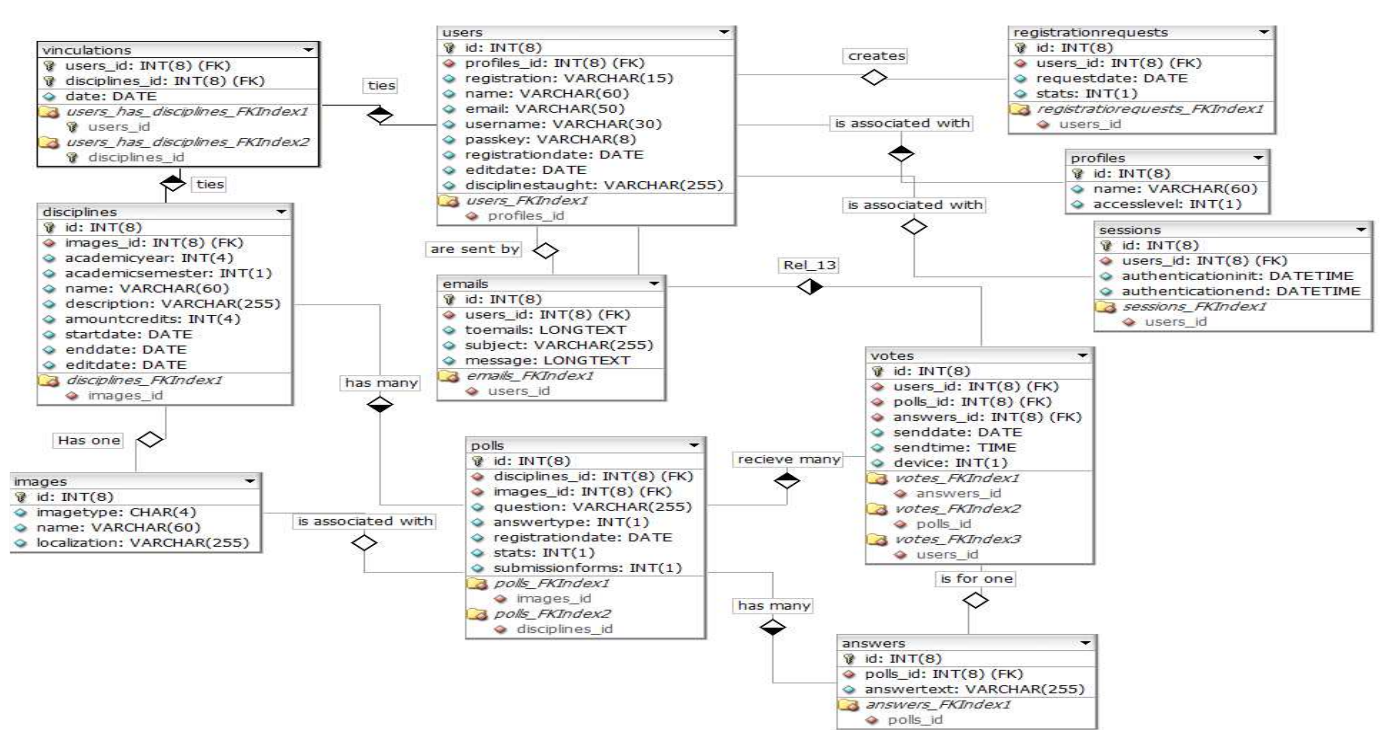

Figura 7 - Modelo relacional da base de dados do EASY-SRA

As classes presentes no diagrama de classes do sistema EASY-SRA (Figura 8) seguiram as tabelas do modelo relacional de base de dados, somente adicionando algumas classes não persistentes como EmailServer, WorksheetGenerator, StatisticsGenerator, Attachment, AttachmmentPoll e AttachmentImage responsáveis, respectivamente, por enviar emails dos usuários, gerar arquivos no formato de planilha eletrônica com os dados de uma votação, gerar estatísticas de votações, representar um anexo tanto de imagem quanto de votação.

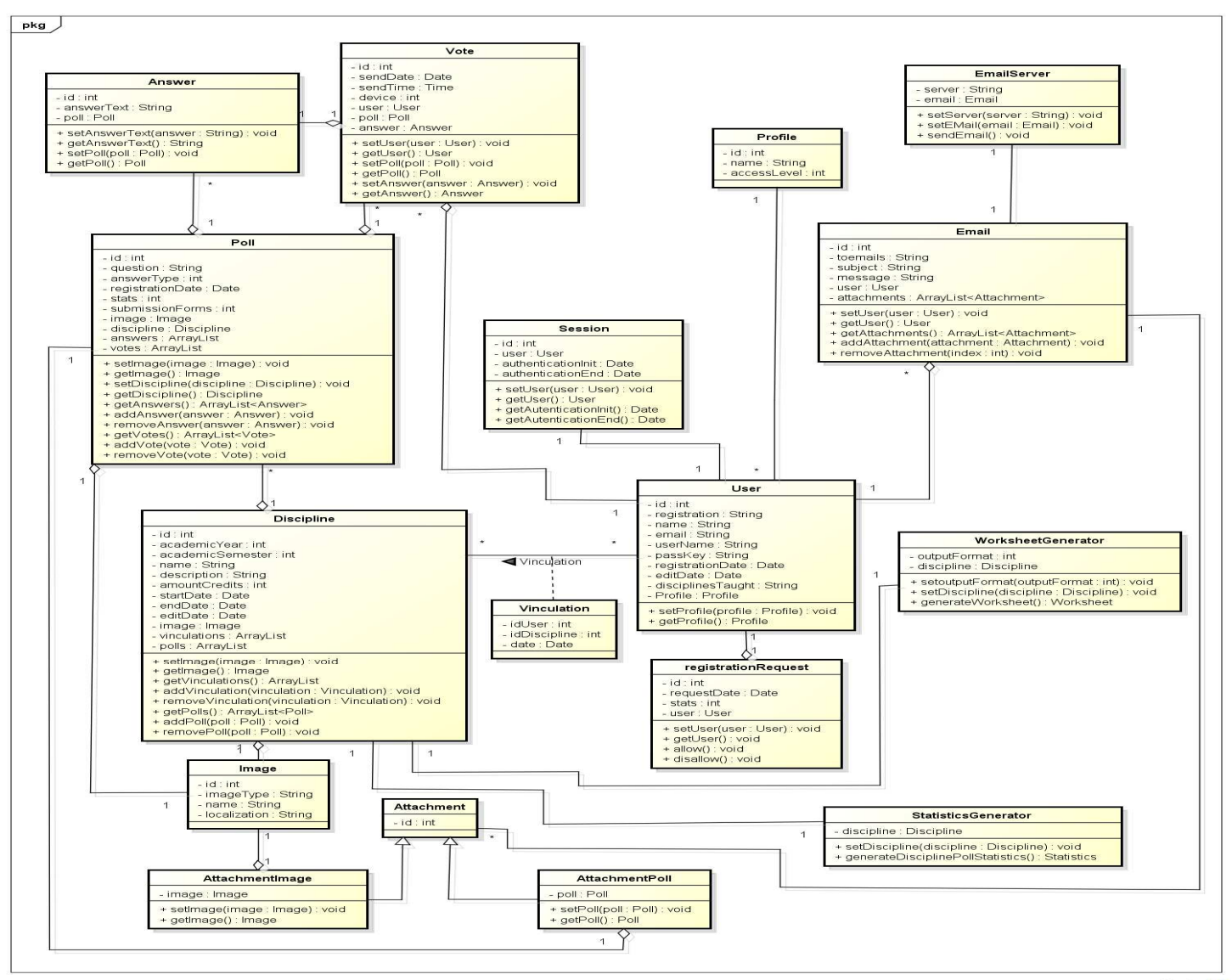

Figura 8 - Diagrama de classes do EASY-SRA 


\subsection{Interfaces}

A figura 9 apresenta a tela inicial para autenticação de um usuário. Nela, o usuário visualiza uma mensagem informando a finalidade do sistema, em paralelo, é conduzido a informar seu login e senha, respectivamente, para ter acesso as funcinalidades do mesmo. A figura 10 mostra a tela inicial de visualização de disciplinas cadastradas no sistema, bem como informações adicionais referentes à: semestre/ano da disciplina, a quantidade de créditos da mesma, data de início das atividades, data de término das atividades e algumas outras ações como, a visualização da lista de estudantes cadastrados na disciplina, a apresentação do relatório da disciplina, a possibilidade de editar e/ou excluir a disciplina.

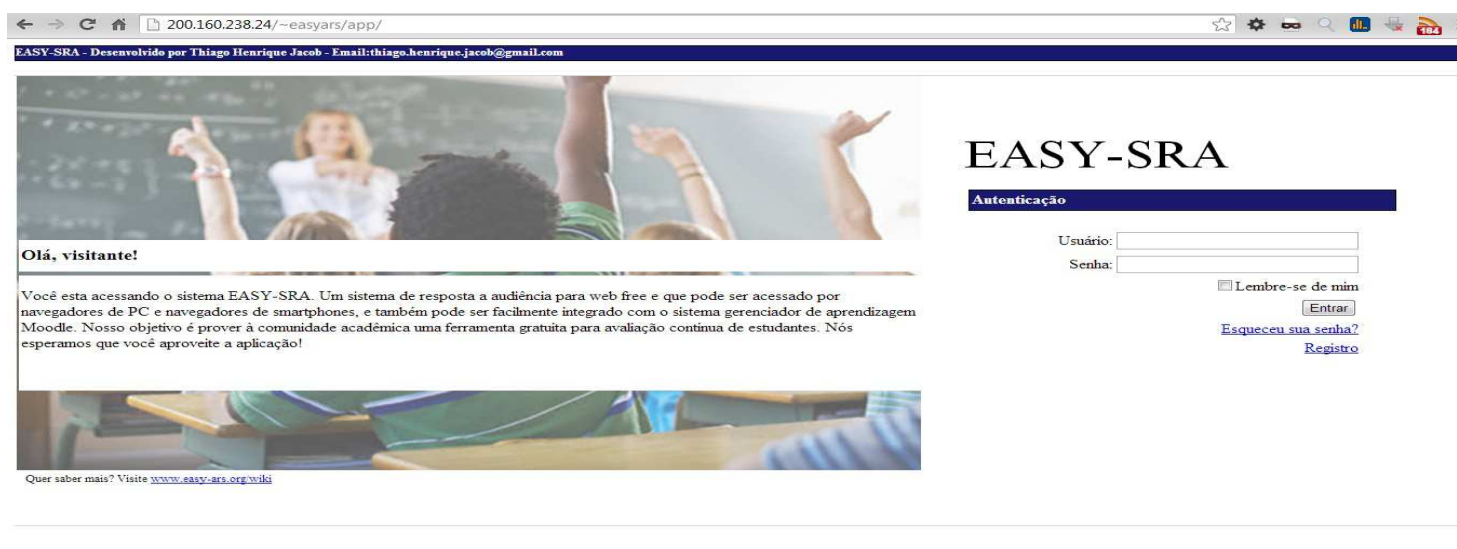

Figura 9 - Interface de autenticação do EASY-SRA

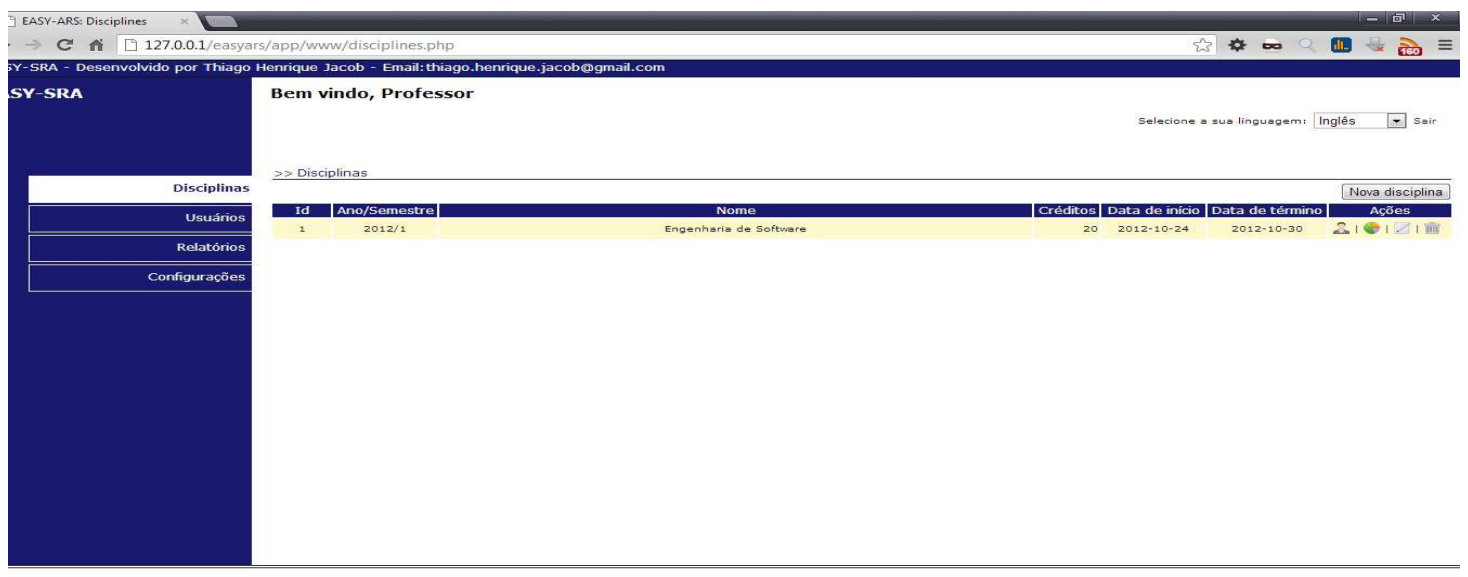

Figura 10 - Interface de visualização de disciplinas do EASY-SRA

\section{Considerações Finais}

Foi apresentado aqui o estudo de uma análise de SRA com o propósito de identificar suas principais funcionalidades para incorporá-las em um novo sistema, o EASY-SRA. Com o objetivo de identificar as funcionalidades mais relevantes em Sistemas de Resposta à Audiência foi proposta uma metodologia baseada nas seguinte fases: estudo bibliográfico sobre SRA; análise comparativa entre sistemas encontrados; e manipulação dos sistemas para registro de suas funcionalidades específicas. A partir desta análise, foram coletados alguns requisitos funcionais e não-funcionais. Assim, foi 
possível reconhecer as características fundamentais de um SRA e identificar as funcionalidades adicionais necessárias ao sistema a ser implementado.

A versão inicial do EASY-SRA está em fase de desenvolvimento e será testada em duas turmas de graduação da UFPB com o propósito de obter dados sobre a eficácia do sistema e aspectos a serem aperfeiçoados. Pretende-se também implementar a integração do EASY-SRA com o sistema gerenciador de aprendizagem MOODLE, bem como integrá-lo a rede social Facebook. Intenciona-se, ainda, disponibilizar o sistema em um site da web para download e criar espaços dentro do sítio para que os interessados no EASY-SRA discutam sobre ele.

\section{Referências Bibliográficas}

BEATTY, I. D.; LEONARD, W. J.; GERACE, W. J.; DUFRESNE, R. J. Question Driven Instruction: Teaching science (Well) with an Audience Response System. 2006.

CALDWELL, J. E. Clickers in the Large Classroom: Current Research and BestPractice Tips. In: CBE-Life Sciences Education. 2007.

GAUCI, S. A.; DANTAS, A. M.; WILlIAMS, D. A.; KEMM, R. E. Promoting student-centered active learning in lectures with a personal response system. In: Advances in Physiology Education, vol. no. 1, p. 60-67. 2009.

KAY, R. H.; LESAGE, A. Examining the benefits and challenges of using audience response systems: A review of the literature, Volume 53, Issue 3, November 2009, p. 819-827.

MANTORO, T.; AYU, M. A.; HABUL, E.; KHASANAH, A. U. Survnvote: A Free Web Based Audience Response System to Support Interactivity in the Classroom. Open Systems (ICOS). In: IEEE Conference, 5-7 December 2010, p.34-39.

SOMMERVILE, I. Engenharia de Software. São Paulo: Pearson Addison Wesley. 8 ed. 2007. 